\title{
New general integral inequalities for quasiconvex functions
}

\section{Cetin Yildiz}

Ataturk University, K. K. Education Faculty, Department of Mathematics, Erzurum, Turkey

Received: 26 July 2016, Accepted: 11 November 2016

Published online: 26 December 2017.

\begin{abstract}
In this paper, by using an integral identity and the Hölder integral inequality we establish several new inequalities for $n-$
\end{abstract} times differentiable mappings that are connected with the quasiconvex functions.

Keywords: Hermite-Hadamard inequality, Hölder inequality, QuasiConvex functions.

\section{Introduction}

Let $f: I \subseteq \mathbb{R} \rightarrow \mathbb{R}$ be a convex function defined on an interval $I$ of real numbers, $a, b \in I$ and $a<b$. The following double inequality is well known in the literature as Hadamard's inequality:

$$
f\left(\frac{a+b}{2}\right) \leq \frac{1}{b-a} \int_{a}^{b} f(x) d x \leq \frac{f(a)+f(b)}{2}
$$

Both inequalities hold in the reversed direction if $f$ is concave.

The inequalities in (1) have become an important cornerstone in mathematical anlysis and optimization. Many uses of these inequalities have been discovered in a variety of settings. Moreover, many inequalities of special means can be obtained for a particular choice of the function $f$. Due to the rich geometrical significance of Hermite-Hadamard inequlity, there is growing literature providing its new proofs, extensions, refinements and generalizations, see for example ([5],[9]-[13]) and the references therein.

Definition 1. A function $f:[a, b] \subset \mathbb{R} \rightarrow \mathbb{R}$ is said to be convex if whenever $x, y \in[a, b]$ and $t \in[0,1]$, the following inequality holds:

$$
f(t x+(1-t) y) \leq t f(x)+(1-t) f(y) .
$$

We say that $f$ is concave if $(-f)$ is convex. This definition has its origins in Jensen's results from [8] and has opened up the most extended, useful and multi-disciplinary domain of mathematics, namely, convex analysis. Convex curves and convex bodies have appeared in mathematical literature since antiquity and there are many important results related to them.

We recall that the notion of quasiconvex functions generalizes the notion of convex functions.

Definition 2. A function $f:[a, b] \subset \mathbb{R} \rightarrow \mathbb{R}$ is said to be quasiconvex on $[a, b]$ if

$$
f(t x+(1-t) y) \leq \max \{f(x), f(y)\}
$$

*Corresponding author e-mail: cetin@ atauni.edu.tr 
for all $x, y \in[a, b]$ and $t \in[0,1]$.

Clearly, any convex function is quasiconvex. Furthermore, there exist quasiconvex functions which are not convex (see [7],[14]). For example, consider the following.

Let $f: \mathbb{R}^{+} \rightarrow \mathbb{R}$

$$
f(x)=\ln x, \quad x \in \mathbb{R}^{+} .
$$

This function is quasiconvex. However $f$ is not a convex function. For other recent results concerning the $n-$ times differentiable functions see [2]- [4],[6],[9],[11],[15]-[17] where further references are given.

In [1], Alomari et. al. proved the following theorems for quasiconvex functions.

Theorem 1. Let $f: I \subset[0, \infty) \rightarrow \mathbb{R}$ be a differentiable mapping on $I^{\circ}$ such that $f^{\prime} \in L[a, b]$, where $a, b \in I$ with $a<b$. If $\left|f^{\prime}\right|$ is an quasi-convex on $[a, b]$, then the following inequality holds.

$$
\left|\frac{f(a)+f(b)}{2}-\frac{1}{b-a} \int_{a}^{b} f(x) d x\right| \leq \frac{b-a}{8}\left[\max \left\{\left|f^{\prime}\left(\frac{a+b}{2}\right)\right|,\left|f^{\prime}(a)\right|\right\}+\max \left\{\left|f^{\prime}\left(\frac{a+b}{2}\right)\right|,\left|f^{\prime}(b)\right|\right\}\right] .
$$

Theorem 2. Let $f: I \subset[0, \infty) \rightarrow \mathbb{R}$ be a differentiable mapping on $I^{\circ}$ such that $f^{\prime} \in L[a, b]$, where $a, b \in I$ with $a<b$. If $\left|f^{\prime}\right|^{p /(p-1)}$ is an quasi-convex on $[a, b]$, for $p>1$, then the following inequality holds:

$$
\begin{aligned}
\left|\frac{f(a)+f(b)}{2}-\frac{1}{b-a} \int_{a}^{b} f(x) d x\right| & \leq \frac{(b-a)}{4(p+1)^{1 / p}}\left[\left(\max \left\{\left|f^{\prime}\left(\frac{a+b}{2}\right)\right|^{p /(p-1)},\left|f^{\prime}(a)\right|^{p /(p-1)}\right\}\right)^{(p-1) / p}\right. \\
& \left.+\left(\max \left\{\left|f^{\prime}\left(\frac{a+b}{2}\right)\right|^{p /(p-1)},\left|f^{\prime}(b)\right|^{p /(p-1)}\right\}\right)^{(p-1) / p}\right]
\end{aligned}
$$

Theorem 3. Let $f: I^{\circ} \subset \mathbb{R} \rightarrow \mathbb{R}$ be a differentiable mapping on $I^{\circ}$ such that $f^{\prime} \in L[a, b]$, where $a, b \in I^{\circ}$ with $a<b$. If $\left|f^{\prime}\right|^{q}$ is an quasi-convex on $[a, b]$, for $p \geq 1$, then the following inequality holds.

$$
\left|\frac{f(a)+f(b)}{2}-\frac{1}{b-a} \int_{a}^{b} f(x) d x\right| \leq \frac{b-a}{8}\left[\left(\max \left\{\left|f^{\prime}\left(\frac{a+b}{2}\right)\right|^{q},\left|f^{\prime}(a)\right|^{q}\right\}\right)^{\frac{1}{q}}+\left(\max \left\{\left|f^{\prime}\left(\frac{a+b}{2}\right)\right|^{q},\left|f^{\prime}(b)\right|^{q}\right\}\right)^{\frac{1}{q}}\right]
$$

The main purpose of the present paper is to prove several new inequalities for quasiconvex functions that are connected with the celebrated Hermite-Hadamard integral inequality.

\section{Main results}

Lemma 1. Let $f:[a, b] \rightarrow \mathbb{R}$ be n-times differentiable functions. If $f^{(n)} \in L[a, b]$, then

$$
\begin{aligned}
\int_{a}^{b} f(t) d t & =\sum_{k=0}^{n-1} \frac{f^{(k)}(a)+(-1)^{k} f^{(k)}(b)}{(k+1) !}\left(\frac{b-a}{2}\right)^{k+1}+(-1)^{n} \frac{(b-a)^{n+1}}{2^{n+1} n !}\left\{\int_{0}^{1}(t-1)^{n} f^{(n)}\left(t \frac{a+b}{2}+(1-t) a\right) d t\right. \\
& \left.+\int_{0}^{1}(1-t)^{n} f^{(n)}\left(t \frac{a+b}{2}+(1-t) b\right) d t\right\}
\end{aligned}
$$

where $n$ natural number, $n \geq 1$. 
Proof. The proof is by mathematical induction. For $n=1$, we have to prove the equality

$$
\frac{f(a)+f(b)}{2}-\frac{1}{b-a} \int_{a}^{b} f(t) d t=\frac{b-a}{4}\left\{\int_{0}^{1}(t-1) f^{\prime}\left(t \frac{a+b}{2}+(1-t) a\right) d t+\int_{0}^{1}(1-t) f^{\prime}\left(t \frac{a+b}{2}+(1-t) b\right) d t\right\} .
$$

Integrating by parts, we have above equality.

Assume that (5) holds for " $n$ " and let us prove it for " $n+1$ ". That is, we have to prove the equality

$$
\begin{aligned}
\int_{a}^{b} f(t) d t & =\sum_{k=0}^{n} \frac{f^{(k)}(a)+(-1)^{k} f^{(k)}(b)}{(k+1) !}\left(\frac{b-a}{2}\right)^{k+1}+(-1)^{n+1} \frac{(b-a)^{n+2}}{2^{n+2}(n+1) !}\left\{\int_{0}^{1}(t-1)^{n+1} f^{(n+1)}\left(t \frac{a+b}{2}+(1-t) a\right) d t\right. \\
& \left.+\int_{0}^{1}(1-t)^{n+1} f^{(n+1)}\left(t \frac{a+b}{2}+(1-t) b\right) d t\right\} .
\end{aligned}
$$

Then, we can write

$$
I=\frac{(b-a)^{n+2}}{2^{n+2}(n+1) !}\left\{\int_{0}^{1}(t-1)^{n+1} f^{(n+1)}\left(t \frac{a+b}{2}+(1-t) a\right) d t+\int_{0}^{1}(1-t)^{n+1} f^{(n+1)}\left(t \frac{a+b}{2}+(1-t) b\right) d t\right\}
$$

and integrating by parts gives

$$
\begin{aligned}
I & =\frac{(b-a)^{n+2}}{2^{n+2}(n+1) !}\left\{\left.(t-1)^{n+1} \frac{f^{(n)}\left(t \frac{a+b}{2}+(1-t) a\right)}{\frac{b-a}{2}}\right|_{0} ^{1}-\frac{n+1}{\frac{b-a}{2}} \int_{0}^{1}(t-1)^{n} f^{(n)}\left(t \frac{a+b}{2}+(1-t) a\right) d t\right\} \\
& +\frac{(b-a)^{n+2}}{2^{n+2}(n+1) !}\left\{\left.(1-t)^{n+1} \frac{f^{(n)}\left(t \frac{a+b}{2}+(1-t) b\right)}{\frac{a-b}{2}}\right|_{0} ^{1}+\frac{n+1}{\frac{a-b}{2}} \int_{0}^{1}(1-t)^{n} f^{(n)}\left(t \frac{a+b}{2}+(1-t) b\right) d t\right\} \\
& =(-1)^{n+2} \frac{(b-a)^{n+1}}{2^{n+1}(n+1) !} f^{(n)}(a)-\frac{(b-a)^{n+1}}{2^{n+1} n !} \int_{0}^{1}(t-1)^{n} f^{(n)}\left(t \frac{a+b}{2}+(1-t) a\right) d t \\
& +\frac{(b-a)^{n+1}}{2^{n+1}(n+1) !} f^{(n)}(b)-\frac{(b-a)^{n+1}}{2^{n+1} n !} \int_{0}^{1}(1-t)^{n} f^{(n)}\left(t \frac{a+b}{2}+(1-t) b\right) d t .
\end{aligned}
$$

Now, using the mathematical induction hypothesis, we get

$$
\frac{1}{(-1)^{n}} \int_{a}^{b} f(t) d t=\frac{1}{(-1)^{n}} \sum_{k=0}^{n-1} \frac{f^{(k)}(a)+(-1)^{k} f^{(k)}(b)}{(k+1) !}\left(\frac{b-a}{2}\right)^{k+1}+(-1)^{n+2} \frac{(b-a)^{n+1}}{2^{n+1}(n+1) !} f^{(n)}(a)+\frac{(b-a)^{n+1}}{2^{n+1}(n+1) !} f^{(n)}(b)-I .
$$

Multiplying the both sides of (7) by $(-1)^{n}$, we obtain

$$
\begin{aligned}
\int_{a}^{b} f(t) d t & =\sum_{k=0}^{n-1} \frac{f^{(k)}(a)+(-1)^{k} f^{(k)}(b)}{(k+1) !}\left(\frac{b-a}{2}\right)^{k+1}+\frac{(b-a)^{n+1}}{2^{n+1}(n+1) !} f^{(n)}(a)+(-1)^{n} \frac{(b-a)^{n+1}}{2^{n+1}(n+1) !} f^{(n)}(b) \\
- & (-1)^{n}\left\{w w \int_{0}^{1}(t-1)^{n+1} f^{(n+1)}\left(t \frac{a+b}{2}+(1-t) a\right) d t+w w \int_{0}^{1}(1-t)^{n+1} f^{(n+1)}\left(t \frac{a+b}{2}+(1-t) b\right) d t\right\} \\
= & \sum_{k=0}^{n} \frac{f^{(k)}(a)+(-1)^{k} f^{(k)}(b)}{(k+1) !}\left(\frac{b-a}{2}\right)^{k+1}+(-1)^{n+1} \frac{(b-a)^{n+2}}{2^{n+2}(n+1) !}\left\{\int_{0}^{1}(t-1)^{n+1} f^{(n+1)}\left(t \frac{a+b}{2}+(1-t) a\right) d t\right. \\
+ & \int_{0}^{1}(1-t)^{n+1} f^{(n+1)}\left(t \frac{a+b}{2}+(1-t) b\right) d t .
\end{aligned}
$$

where $w w=\frac{(b-a)^{n+2}}{2^{n+2}(n+1) !}$. Thus, the identity (6) and the lemma is proved. 
Theorem 4. For $n \geq 1$, let $f:[a, b] \subset \mathbb{R} \rightarrow \mathbb{R}$ be $n$-times differentiable function and $0 \leq a<b$. If $f^{(n)} \in L[a, b]$ and $\left|f^{(n)}\right|$ is quasiconvex on $[a, b]$, then the following inequality holds:

$$
\begin{aligned}
\left|\int_{a}^{b} f(t) d t-\sum_{k=0}^{n-1} \frac{f^{(k)}(a)+(-1)^{k} f^{(k)}(b)}{(k+1) !}\left(\frac{b-a}{2}\right)^{k+1}\right| & \leq \frac{(b-a)^{n+1}}{2^{n+1}(n+1) !}\left[\max \left\{\left|f^{(n)}(a)\right|,\left|f^{(n)}\left(\frac{a+b}{2}\right)\right|\right\}\right. \\
& \left.+\max \left\{\left|f^{(n)}\left(\frac{a+b}{2}\right)\right|,\left|f^{(n)}(b)\right|\right\}\right] .
\end{aligned}
$$

Proof. From Lemma 1, it follows that

$$
\begin{aligned}
\left|\int_{a}^{b} f(t) d t-\sum_{k=0}^{n-1} \frac{f^{(k)}(a)+(-1)^{k} f^{(k)}(b)}{(k+1) !}\left(\frac{b-a}{2}\right)^{k+1}\right| \leq & \frac{(b-a)^{n+1}}{2^{n+1} n !}\left\{\int_{0}^{1}(1-t)^{n}\left|f^{(n)}\left(t \frac{a+b}{2}+(1-t) a\right)\right| d t\right. \\
& \left.+\int_{0}^{1}(1-t)^{n}\left|f^{(n)}\left(t \frac{a+b}{2}+(1-t) b\right)\right| d t\right\}
\end{aligned}
$$

Since $\left|f^{(n)}\right|$ is quasi-convex on $[a, b]$, we obtain

$$
\begin{aligned}
\left|\int_{a}^{b} f(t) d t-\sum_{k=0}^{n-1} \frac{f^{(k)}(a)+(-1)^{k} f^{(k)}(b)}{(k+1) !}\left(\frac{b-a}{2}\right)^{k+1}\right| & \leq \frac{(b-a)^{n+1}}{2^{n+1}(n+1) !}\left[\max \left\{\left|f^{(n)}(a)\right|,\left|f^{(n)}\left(\frac{a+b}{2}\right)\right|\right\}\right. \\
& \left.+\max \left\{\left|f^{(n)}\left(\frac{a+b}{2}\right)\right|,\left|f^{(n)}(b)\right|\right\}\right]
\end{aligned}
$$

This completes the proof.

Corollary 1. Let $f$ as in Theorem 4, if in addition

(1) $\left|f^{(n)}\right|$ is increasing, then we have

$$
\left|\int_{a}^{b} f(t) d t-\sum_{k=0}^{n-1} \frac{f^{(k)}(a)+(-1)^{k} f^{(k)}(b)}{(k+1) !}\left(\frac{b-a}{2}\right)^{k+1}\right| \leq \frac{(b-a)^{n+1}}{2^{n+1}(n+1) !}\left[\left|f^{(n)}\left(\frac{a+b}{2}\right)\right|+\left|f^{(n)}(b)\right|\right]
$$

(2) $\left|f^{(n)}\right|$ is decreasing, then we have

$$
\left|\int_{a}^{b} f(t) d t-\sum_{k=0}^{n-1} \frac{f^{(k)}(a)+(-1)^{k} f^{(k)}(b)}{(k+1) !}\left(\frac{b-a}{2}\right)^{k+1}\right| \leq \frac{(b-a)^{n+1}}{2^{n+1}(n+1) !}\left[\left|f^{(n)}(a)\right|+\left|f^{(n)}\left(\frac{a+b}{2}\right)\right|\right] .
$$

Remark. Under conditions of Theorem 4, if we choose $\mathrm{n}=1$; then we obtain inequality (2).

Theorem 5. Let $f:[a, b] \subset \mathbb{R} \rightarrow \mathbb{R}$ be $n$-times differentiable and $0 \leq a<b$. If $f^{(n)} \in L[a, b]$ and $\left|f^{(n)}\right|^{q}$ is quasiconvex on $[a, b]$, then the following inequality holds.

$$
\begin{aligned}
\left|\int_{a}^{b} f(t) d t-\sum_{k=0}^{n-1} \frac{f^{(k)}(a)+(-1)^{k} f^{(k)}(b)}{(k+1) !}\left(\frac{b-a}{2}\right)^{k+1}\right| & \leq \frac{(b-a)^{n+1}}{2^{n+1} n !}\left(\frac{1}{n p+1}\right)^{\frac{1}{p}}\left[\left(\max \left\{\left|f^{(n)}(a)\right|^{q},\left|f^{(n)}\left(\frac{a+b}{2}\right)\right|^{q}\right\}\right)^{\frac{1}{q}}\right. \\
& \left.+\left(\max \left\{\left|f^{(n)}\left(\frac{a+b}{2}\right)\right|^{q},\left|f^{(n)}(b)\right|^{q}\right\}\right)^{\frac{1}{q}}\right]
\end{aligned}
$$

where $q>1$. 
Proof. From Lemma 1 and the Hölder integral inequality, we obtain

$$
\begin{aligned}
\left|\int_{a}^{b} f(t) d t-\sum_{k=0}^{n-1} \frac{f^{(k)}(a)+(-1)^{k} f^{(k)}(b)}{(k+1) !}\left(\frac{b-a}{2}\right)^{k+1}\right| & \leq \frac{(b-a)^{n+1}}{2^{n+1} n !}\left\{\left(\int_{0}^{1}(1-t)^{n p} d t\right)^{\frac{1}{p}}\left(\int_{0}^{1}\left|f^{(n)}\left(t \frac{a+b}{2}+(1-t) a\right)\right|^{q} d t\right)^{\frac{1}{q}}\right. \\
& \left.+\left(\int_{0}^{1}(1-t)^{n p} d t\right)^{\frac{1}{p}}\left(\int_{0}^{1}\left|f^{(n)}\left(t \frac{a+b}{2}+(1-t) b\right)\right|^{q} d t\right)^{\frac{1}{q}}\right\} .
\end{aligned}
$$

Since $\left|f^{(n)}\right|^{q}$ is quasiconvex on $[a, b]$, for $q>1$, then

$$
\begin{aligned}
\left|\int_{a}^{b} f(t) d t-\sum_{k=0}^{n-1} \frac{f^{(k)}(a)+(-1)^{k} f^{(k)}(b)}{(k+1) !}\left(\frac{b-a}{2}\right)^{k+1}\right| & \leq \frac{(b-a)^{n+1}}{2^{n+1} n !}\left(\frac{1}{n p+1}\right)^{\frac{1}{p}}\left[\left(\max \left\{\left|f^{(n)}(a)\right|^{q},\left|f^{(n)}\left(\frac{a+b}{2}\right)\right|^{q}\right\}\right)^{\frac{1}{q}}\right. \\
& \left.+\left(\max \left\{\left|f^{(n)}\left(\frac{a+b}{2}\right)\right|^{q},\left|f^{(n)}(b)\right|^{q}\right\}\right)^{\frac{1}{q}}\right]
\end{aligned}
$$

which completes the proof.

Corollary 2. Let $f$ as in Theorem 5, if in addition

(1) $\left|f^{(n)}\right|^{q}$ is increasing, then we have

$$
\left|\int_{a}^{b} f(t) d t-\sum_{k=0}^{n-1} \frac{f^{(k)}(a)+(-1)^{k} f^{(k)}(b)}{(k+1) !}\left(\frac{b-a}{2}\right)^{k+1}\right| \leq \frac{(b-a)^{n+1}}{2^{n+1} n !}\left(\frac{1}{n p+1}\right)^{\frac{1}{p}}\left[\left|f^{(n)}\left(\frac{a+b}{2}\right)\right|+\left|f^{(n)}(b)\right|\right] .
$$

(2) $\left|f^{(n)}\right|^{q}$ is decreasing, then we have

$$
\left|\int_{a}^{b} f(t) d t-\sum_{k=0}^{n-1} \frac{f^{(k)}(a)+(-1)^{k} f^{(k)}(b)}{(k+1) !}\left(\frac{b-a}{2}\right)^{k+1}\right| \leq \frac{(b-a)^{n+1}}{2^{n+1} n !}\left(\frac{1}{n p+1}\right)^{\frac{1}{p}}\left[\left|f^{(n)}(a)\right|+\left|f^{(n)}\left(\frac{a+b}{2}\right)\right|\right] .
$$

Remark. Under conditions of Theorem 5, if we choose $\mathrm{n}=1$; then we obtain inequality (3).

Theorem 6. For $n \geq 1$, let $f:[a, b] \subset \mathbb{R} \rightarrow \mathbb{R}$ be $n$-times differentiable and $0 \leq a<b$. If $f^{(n)} \in L[a, b]$ and $\left|f^{(n)}\right|^{q}$ is quasiconvex on $[a, b]$, for $q \geq 1$, then the following inequality holds.

$$
\begin{aligned}
\left|\int_{a}^{b} f(t) d t-\sum_{k=0}^{n-1} \frac{f^{(k)}(a)+(-1)^{k} f^{(k)}(b)}{(k+1) !}\left(\frac{b-a}{2}\right)^{k+1}\right| & \leq \frac{(b-a)^{n+1}}{2^{n+1}(n+1) !}\left[\left(\max \left\{\left|f^{(n)}(a)\right|^{q},\left|f^{(n)}\left(\frac{a+b}{2}\right)\right|^{q}\right\}\right)^{\frac{1}{q}}\right. \\
& \left.+\left(\max \left\{\left|f^{(n)}\left(\frac{a+b}{2}\right)\right|^{q},\left|f^{(n)}(b)\right|^{q}\right\}\right)^{\frac{1}{q}}\right] .
\end{aligned}
$$

Proof. From Lemma 1 and using the well known power-mean integral inequality, we have

$$
\begin{aligned}
& \left|\int_{a}^{b} f(t) d t-\sum_{k=0}^{n-1} \frac{f^{(k)}(a)+(-1)^{k} f^{(k)}(b)}{(k+1) !}\left(\frac{b-a}{2}\right)^{k+1}\right| \leq \frac{(b-a)^{n+1}}{2^{n+1} n !}\left\{\int_{0}^{1}(1-t)^{n}\left|f^{(n)}\left(t \frac{a+b}{2}+(1-t) a\right)\right| d t\right. \\
& \left.+\int_{0}^{1}(1-t)^{n}\left|f^{(n)}(w t+(1-t) b)\right| d t\right\} \leq \frac{(b-a)^{n+1}}{2^{n+1} n !}\left\{\left(\int_{0}^{1}(1-t)^{n} d t\right)^{1-\frac{1}{q}}\left(\int_{0}^{1}(1-t)^{n}\left|f^{(n)}(w t+(1-t) a)\right|^{q} d t\right)^{\frac{1}{q}}\right. \\
& \left.+\left(\int_{0}^{1}(1-t)^{n} d t\right)^{1-\frac{1}{q}}\left(\int_{0}^{1}(1-t)^{n}\left|f^{(n)}\left(t \frac{a+b}{2}+(1-t) b\right)\right|^{q} d t\right)^{\frac{1}{q}}\right\}
\end{aligned}
$$


where $t \frac{a+b}{2}$. Since $\left|f^{(n)}\right|^{q}$ is quasiconvex on $[a, b]$, for $q \geq 1$, then we obtain

$$
\begin{aligned}
\left|\int_{a}^{b} f(t) d t-\sum_{k=0}^{n-1} \frac{f^{(k)}(a)+(-1)^{k} f^{(k)}(b)}{(k+1) !}\left(\frac{b-a}{2}\right)^{k+1}\right| & \leq \frac{(b-a)^{n+1}}{2^{n+1}(n+1) !}\left[\left(\max \left\{\left|f^{(n)}(a)\right|^{q},\left|f^{(n)}\left(\frac{a+b}{2}\right)\right|^{q}\right\}\right)^{\frac{1}{q}}\right. \\
& \left.+\left(\max \left\{\left|f^{(n)}\left(\frac{a+b}{2}\right)\right|^{q},\left|f^{(n)}(b)\right|^{q}\right\}\right)^{\frac{1}{q}}\right]
\end{aligned}
$$

which completes the proof.

Remark. Under conditions of Theorem 6, if we choose $n=1$; then we obtain inequality (4).

\section{Conclusions}

In this study, we presented some generalized Hermite type inequalities for the mappings whose derivatives are quasiconvex functions are established. A further study could be assess weighted versions of these inequalities.

\section{Competing interests}

The authors declare that they have no competing interests.

\section{Authors' contributions}

All authors have contributed to all parts of the article. All authors read and approved the final manuscript.

\section{References}

[1] M. Alomari, M. Darus and U.S. Kırmaci, Refinements of Hadamard-type inequalities for quasi-convex functions with applications to trapezoidal formula and to special means, Comp. Math. Appl., 59 (2010), 225-232.

[2] S.-P. Bai, S.-H. Wang and F. Qi, Some Hermite-Hadamard type inequalities for $n$-time differentiable $(\alpha, m)$-convex functions, Jour. of Ineq. and Appl., 2012, 2012:267.

[3] P. Cerone, S.S. Dragomir and J. Roumeliotis, Some Ostrowski type inequalities for $n$-time differentiable mappings and applications, Demonstratio Math., 32 (4) (1999), 697-712.

[4] P. Cerone, S.S. Dragomir and J. Roumeliotis and J. Šunde, A new generalization of the trapezoid formula for $n$-time differentiable mappings and applications, Demonstratio Math., 33 (4) (2000), 719-736.

[5] S.S. Dragomir and C.E.M. Pearce, Selected Topics on Hermite-Hadamard Inequalities and Applications, RGMIA Monographs, Victoria University, 2000. Online:[http://www.staxo.vu.edu.au/RGMIA/monographs/hermite hadamard.html].

[6] D. Y. Hwang, Some Inequalities for $n$-time Differentiable Mappings and Applications, Kyung. Math. Jour., 43 (2003), $335-343$.

[7] D.A. Ion, Some estimates on the Hermite-Hadamard inequalities through quasi-convex functions, Annals of University of Craiova, Math. Comp. Sci. Ser., 34 (2007), 82-87.

[8] J. L. W. V. Jensen, On konvexe funktioner og uligheder mellem middlvaerdier, Nyt. Tidsskr. Math. B., 16, 49-69, 1905.

[9] W.-D. Jiang, D.-W. Niu, Y. Hua and F. Qi, Generalizations of Hermite-Hadamard inequality to $n$-time differentiable function which are $s$-convex in the second sense, Analysis (Munich), 32 (2012), 209-220

[10] U.S. Kırmacı, M.K. Bakula, M.E. Özdemir and J. Pećarić, Hadamard-type inequalities for s-convex functions, Appl. Math. and Comp., 193(2007), 26-35.

[11] M. E. Özdemir, Ç. Yıldız, New Inequalities for $n$-time differentiable functions, Arxiv:1402.4959v1. 
[12] M.E. Özdemir, Ç. Yıldız, New Inequalities for Hermite-Hadamard and Simpson Type with Applications, Tamkang J. of Math., 44, 2, 209-216, 2013.

[13] M. E. Özdemir, Ç. Yıldız, A. O. Akdemir and E. Set, New Inequalities of Hadamard Type for Quasi-Convex Functions, AIP Conference Proceedings, 1470, 99-101 (2012); doi: 10.1063/1.4747649.

[14] J.E. Pečarić, F. Porschan and Y.L. Tong, Convex Functions, Partial Orderings, and Statistical Applications, Academic Press Inc., 1992.

[15] Ç. Yıldız, New Inequalities of the Hermite-Hadamard type for $n$-time differentiable functions which are quasiconvex, Jour. Math. Ineq., (10) (3) (2016), 703-711.

[16] Ç. Yıldız, M.E. Özdemir, New generalized inequalities of Hermite-Hadamard type for quasi-convex functions, AIP Conference Proceedings, 1726, 020053-1-020053-4 (2016); doi: 10.1063/1.4945879.

[17] S.H. Wang, B.-Y. Xi and F. Qi, Some new inequalities of Hermite-Hadamard type for $n$-time differentiable functions which are m-convex, Analysis (Munich), 32 (2012), 247-262. 